\title{
The relation between A-harmonic operator and A-Dirac system
}

\section{Zhanlei Wang and Shuhong Chen*}

\section{"Correspondence:} shiny0320@163.com School of Mathematics and Statistics, Minnan Normal University, Zhangzhou, Fujian 363000, China

\begin{abstract}
In this paper, we show how an A-harmonic operator arises from Dirac systems under controllable growth condition. By the method of removable singularities for solutions to the A-Dirac system with controllable growth conditions, we establish the fact that an A-harmonic operator is a real part of the corresponding A-Dirac systems.
\end{abstract}

Keywords: A-harmonic operator; A-Dirac system; Caccioppoli estimate; controllable growth condition

\section{Introduction}

In this paper, we study the relation between an A-harmonic operator and A-Dirac systems under controllable growth conditions. The equations defined by the A-harmonic operator are

$$
-\operatorname{div} A(x, \nabla u)=f(x, \nabla u)
$$

where

$$
A(x, \xi): \Omega \times \mathbb{R}^{n} \rightarrow \mathbb{R}^{n}
$$

$x \rightarrow A(x, \xi)$ is measurable for all $\xi$, and $\xi \rightarrow A(x, \xi)$ is continuous for a.e. $x \in \Omega$. Further assume that $A(x, \xi)$ satisfies the following structure conditions with $p>1$ :

$$
\begin{aligned}
& \langle A(x, \xi), \xi\rangle \geq|\xi|^{p}, \\
& |A(x, \xi)| \leq a|\xi|^{p-1}
\end{aligned}
$$

for some constant $a>0$, and $p$ represents an exponent throughout the paper. The inhomogeneity $f(x, \xi)$ satisfies the following controllable growth condition:

$$
f(x, \nabla u) \leq|\nabla u|^{p\left(1-\frac{1}{s}\right)}+1
$$

where $s=\frac{n p}{n-p}$ for $n>p$; $s$ is any exponent for $n=p$.

\section{Springer}

(0) 2013 Wang and Chen; licensee Springer. This is an Open Access article distributed under the terms of the Creative Commons Attribution License (http://creativecommons.org/licenses/by/2.0), which permits unrestricted use, distribution, and reproduction in any medium, provided the original work is properly cited. 
Definition 1.1 We call a function $u \in W_{\mathrm{loc}}^{1, p}(\Omega)$ a weak solution to (1.1) under the structure conditions (1.3) and (1.4) if the equality

$$
\int_{\Omega}\langle A(x, \nabla u), \nabla \phi\rangle d x=\int_{\Omega} f(x, \nabla u) \phi d x
$$

holds for all $\phi \in W^{1, p}(\Omega)$ with compact support.

The A-Dirac systems in current paper are of the form

$$
-D \tilde{A}(x, D u)=f(x, D u) .
$$

The main purpose of this paper is to show that under controllable growth condition, an equation defined by the A-harmonic operator is a real part of the corresponding A-Dirac system. In order to obtain the desired result, we use the method of removability theorems, which proved that under suitable condition, a result concerning removable singularities for equations defined by the A-harmonic operator satisfying the Lipschitz condition or of bounded mean oscillation extends to Clifford-valued solutions to the corresponding Dirac equation. Further discussion on nonlinear Dirac equations can be found in [1-9] and their references.

The method of removability theorems was introduced by Abreu-Blaya et al. in [1], where they showed that $r^{n} \omega(r)$-Hausdorff measure sets of monogenic functions with modulus of continuity $\omega(r)$ can be removed. The results were extended to Hölder continuous analytic functions [10] by Kaufman and Wu immediately. And then, Koskela and Martio [11] established that in Hölder and bounded mean oscillation classes, the sets satisfying a certain geometric condition related to Minkowski dimension of an A-harmonic function can be removed. In terms of Hausdorff dimension, a precise condition for removable sets of A-harmonic functions in the case of Hölder continuity exists [12]. The results were generalized [9] to the A-Dirac equation satisfying a certain oscillation condition. In the current paper, we further extend the results in [9] to discover the relation between the inhomogeneity A-harmonic equations and the inhomogeneity A-Dirac equations under controllable growth condition and obtain the main result as follows. It implies that under suitable condition, the solutions to the A-harmonic equation under controllable growth condition in fact is a real part of weak solutions to the corresponding A-Dirac systems.

Theorem 1.1 Let E be a relatively closed subset of $\Omega$. Suppose that $u \in L_{\mathrm{loc}}^{p}(\Omega)$ has distributional first derivatives in $\Omega, u$ is a solution to the scalar part of A-Dirac equation (1.6) under controllable growth condition in $\Omega \backslash E$, and $u$ is of $p$, $k$-oscillation in $\Omega \backslash E$. Iffor each compact subset $K$ of $E$

$$
\int_{K(1) \backslash K} d(x, K)^{p(k-1)-k}<\infty,
$$

then $u$ extends to a solution of the A-Dirac equation in $\Omega$.

\section{A-Dirac operator}

In this section, we introduce an A-Dirac operator. In order to definite the A-Dirac operator, we should present the definition and notations about Clifford algebra at first [9]. 
We write $\mathcal{U}_{n}$ for the real universal Clifford algebra over $R^{n}$. The Clifford algebra is generated over $R$ by the basis of reduced products

$$
\left\{e_{1}, e_{2}, \ldots, e_{n}, e_{1} e_{2}, \ldots, e_{1} \cdots e_{n}\right\}
$$

where $\left\{e_{1}, e_{2}, \ldots, e_{n}\right\}$ is an orthogonal basis of $R^{n}$ with the relation $e_{i} e_{j}+e_{j} e_{i}=-2 \delta_{i j}$. We write $e_{0}$ for the identity. The dimension of $\mathcal{U}_{n}$ is $R^{2^{n}}$, which implies an increasing tower $R \subset C \subset H \subset \mathcal{U}_{n} \subset \cdots$. The Clifford algebra $\mathcal{U}_{n}$ is a graded algebra as $\mathcal{U}_{n}=\bigoplus_{l} \mathcal{U}_{n}^{l}$, where $\mathcal{U}_{n}^{l}$ are those elements whose reduced Clifford products have length $l$.

For $A \subset \mathcal{U}_{n}, S c(A)$ denotes the scalar part of $A$, that is, the coefficient of the element $e_{0}$, where $\Omega \subset R^{n}$ is a connected and open set with boundary $\partial \Omega$. A Clifford-valued function $u: \Omega \rightarrow \mathcal{U}_{n}$ can be written as $u=\sum_{\alpha} u_{\alpha} e_{\alpha}$, where each $u_{\alpha}$ is real-valued and $e_{\alpha}$ are reduced products. The norm used here is given by $\left|\sum_{\alpha} u_{\alpha} e_{\alpha}\right|=\left(\sum_{\alpha} u_{\alpha}^{2}\right)^{1 / 2}$. This norm is sub-multiplicative, $|A B| \leq C|A B|$.

The Dirac operator used here is

$$
D=\sum_{j=1}^{n} e_{j} \frac{\partial}{\partial x_{j}}
$$

Also, $D^{2}=-\triangle$. Here $\triangle$ is the Laplace operator which operates only on coefficients. A function is monogenic when $D u=0$.

$Q$ is a cube in $\Omega$ with volume $|Q|$ throughout the paper. We write $\sigma Q$ for the cube with the same center as $Q$ and with sidelength $\sigma$ times that of $Q$. For $q>0$, we write $L^{q}\left(\Omega, \mathcal{U}_{n}\right)$ for the space of Clifford-valued functions in $\Omega$ whose coefficients belong to the usual $L^{q}(\Omega)$ space. Also, $W^{1, q}\left(\Omega, \mathcal{U}_{n}\right)$ is the space of Clifford-valued functions in $\Omega$ whose coefficients as well as their first distributional derivatives are in $L^{q}(\Omega)$. We also write $L_{\text {loc }}^{q}\left(\Omega, \mathcal{U}_{n}\right)$ for $\cap L^{q}\left(\Omega^{\prime}, \mathcal{U}_{n}\right)$, where the intersection is over all $\Omega^{\prime}$ compactly contained in $\Omega$. We similarly write $W_{\text {loc }}^{1, q}\left(\Omega, \mathcal{U}_{n}\right)$. Moreover, we write $\mathcal{M}_{\Omega}=\left\{u: \Omega \rightarrow \mathcal{U}_{n} \mid D u=0\right\}$ for the space of monogenic functions in $\Omega$.

Furthermore, we define the Dirac Sobolev space

$$
W^{D, p}(\Omega)=\left\{\left.u \in \mathcal{U}_{n}\left|\int_{\Omega}\right| u\right|^{p}+\int_{\Omega}|D u|^{p}<\infty\right\}
$$

The local space $W_{\text {loc }}^{D, p}$ is similarly defined. Notice that if $u$ is monogenic, then $u \in L^{p}(\Omega)$ if and only if $u \in W^{D, p}(\Omega)$. Also, it is immediate that $W^{1, p}(\Omega) \subset W^{D, p}(\Omega)$.

Under such definitions and notations, we can introduce the operator of A-Dirac. Define linear isomorphism $\theta: R^{n} \rightarrow \mathcal{U}_{n}^{1}$ by

$$
\theta\left(w_{1}, \ldots, w_{n}\right)=\sum_{i=1}^{n} w_{i} e_{i}
$$

For $x, y \in \mathbb{R}^{n}$, we have

$$
\begin{aligned}
& -\operatorname{Re}(\theta(x) \theta(y))=\langle x, y\rangle, \\
& |\theta(x)|=|x| .
\end{aligned}
$$


Here $\tilde{A}(x, \xi): \Omega \times \mathcal{U}_{1} \rightarrow \mathcal{U}_{1}$ is defined by

$$
\tilde{A}(x, \xi)=\theta A\left(x, \theta^{-1} \xi\right)
$$

which means that (1.5) is equivalent to

$$
\int_{\Omega} \operatorname{Re}(\theta A(x, \nabla u) \theta(\nabla \phi)) d x=\int_{\Omega} \operatorname{Re}(\tilde{A}(x, D u) D \phi) d x=\int_{\Omega} f(x, \nabla u) \phi d x .
$$

For the Clifford conjugation $\overline{\left(e_{j 1} \cdots e_{j l}\right)}=(-1)^{l} e_{j l} \cdots e_{j 1}$, we define a Clifford-valued inner product as $\bar{\alpha} \beta$. Moreover, the scalar part of this Clifford inner product $\operatorname{Re}(\bar{\alpha} \beta)$ is the usual inner product in $\mathbb{R}^{2^{n}},\langle\alpha, \beta\rangle$, when $\alpha$ and $\beta$ are identified as vectors.

For convenience, we replace $\tilde{A}$ with $A$, recast the structure equations above and define the operator

$$
A(x, \xi): \Omega \times \mathcal{U}_{n} \rightarrow \mathcal{U}_{n}
$$

where $A$ preserves the grading of the Clifford algebra, $x \rightarrow A(x, \xi)$ is measurable for all $\xi$, and $\xi \rightarrow A(x, \xi)$ is continuous for a.e. $x \in \Omega$. Furthermore, here $A(x, \xi)$ satisfies the structure conditions with $p>1$,

$$
\begin{aligned}
& \operatorname{Re}(\overline{A(x, \xi)} \xi) \geq|\xi|^{p} \\
& |A(x, \xi)| \leq a|\xi|^{p-1}
\end{aligned}
$$

for some constant $a>0$. We can definite the weak solution of equation (1.6) as follows.

Definition 2.1 A Clifford-valued function $u \in W_{\text {loc }}^{D, p}\left(\Omega, \mathcal{U}_{n}^{k}\right)$, for $k=0,1,2, \ldots, n$, is a weak solution to (1.6) under structure conditions (2.10) and (2.11), and further assume that the inhomogeneity term $f(x, D u)$ satisfies the following controllable growth condition:

$$
f(x, D u) \leq|D u|^{p\left(1-\frac{1}{s}\right)}+1,
$$

where $s=\frac{n p}{n-p}$ for $n>p$; $s$ is any exponent for $n=p$.

For all $\phi \in W^{1, p}\left(\Omega, \mathcal{U}_{n}^{k}\right)$ with compact support we have

$$
\int_{\Omega} \overline{A(x, D u)} D \phi d x=\int_{\Omega} \overline{f(x, D u)} \phi d x
$$

Notice that when $A$ is identity, then the homogeneity part of (2.13)

$$
\int_{\Omega} \overline{A(x, D u)} D \phi d x=0
$$

is the Clifford Laplacian. Moreover, these equations generalize the important case of the $p$-Dirac equation

$$
D\left(|D u|^{n-2} D u\right)=0 .
$$

Here $A(x, \xi)=|\xi|^{p-2} \xi$. 
These equations were introduced and their conformal invariance was studied in [7].

Furthermore, when $u$ is a real-valued function, (2.14) implies that $A(x, \nabla u)$ is a harmonic field, and locally there exists a harmonic function $H$ such that $A(x, \nabla u)=\nabla H$. If $A(x, \xi)$ is invertible, then $\nabla u=A^{-1}(x, \nabla H)$. Hence, the regularity of $A$ implies the regularity of the solution $u$.

In general, A-harmonic functions do not have such regularity. This suggests the study of the scalar part of system (2.13) in general. Thus a Caccioppoli estimate for solutions to the scalar part of (2.13) is necessary.

\section{The proof of main results}

In this section, we establish the main results. Thus, a suitable Caccioppoli estimate for solutions to (2.13) is necessary.

Theorem 3.1 Let $u$ be a solution to the scalar part of (1.6) defined by (2.13), and let $Q$ be a cube with $\sigma Q \subset \Omega$, where $\sigma>1$. Then

$$
\int_{Q}|D u|^{p} \leq C(\varepsilon)|Q|^{-p / n} \int_{\sigma Q}\left|u-u_{\sigma Q}\right|^{p}+C(\varepsilon)\left(\int_{Q}\left(|D u|^{p}+1\right) d x\right)^{p(s-1) / s(p-1)} .
$$

Proof Let $\eta \in C_{\infty}^{0}(\Omega)$ be a standard cut-off function, $\eta>0, \eta \equiv 1$ in $Q$. Choose $\phi=$ $\left(u-u_{\sigma Q}\right) \eta^{p}$ as a test function in (2.13). Then $D \phi=p \eta^{p-1}(D \eta)\left(u-u_{\sigma Q}\right)+\eta^{p} D u$. Using the structure conditions (2.10) and (2.11),

$$
\begin{aligned}
& \int_{\Omega} f(x, D u)\left(u-u_{\sigma Q}\right) \eta^{p} d x \\
& \quad=\int_{\Omega} \operatorname{Re}\left(\overline{A(x, D u)}\left(p \eta^{p-1}(D \eta)\left(u-u_{\sigma Q}\right)+\eta^{p} D u\right)\right) d x,
\end{aligned}
$$

which means that

$$
\begin{aligned}
\int_{\Omega}|D u|^{p} \eta^{p} d x \leq & a p \int_{\Omega}|D u|^{p-1}\left|u-u_{\sigma Q}\right||D \eta||\eta|^{p-1} d x \\
& +\int_{\Omega} f(x, D u)\left(u-u_{\sigma Q}\right) \eta^{p} d x .
\end{aligned}
$$

Using Hölder's inequality and (2.11), we have

$$
\begin{aligned}
\int_{\Omega}|D u|^{p} \eta^{p} \leq & C(p, a)\left(\int_{\Omega}\left|u-u_{\sigma Q}\right|^{p}|D \eta|^{p}\right)^{1 / p}\left(\int_{\Omega}|D u|^{p} \eta^{p}\right)^{(p-1) / p} \\
& +\int_{\Omega}|f(x, D u)|\left|u-u_{\sigma Q} \| \eta\right|^{p} \\
= & I_{1}+I_{2} .
\end{aligned}
$$

Using Young's inequality, we get

$$
I_{1} \leq \varepsilon \int_{\Omega}|D u|^{p}|\eta|^{p}+C(\varepsilon, a, p) \int_{\Omega}\left|u-u_{\sigma Q}\right|^{p}|D \eta|^{p} .
$$


Using (2.12) and then the Sobolev embedding theorem yields

$$
\begin{aligned}
I_{2} & \leq \int_{\Omega}\left|u-u_{\sigma Q}\right| \eta\left(|D u|^{p(1-1 / s)}+1\right) \eta^{p-1} d x \\
& \leq\left(\int_{\Omega}\left|u-u_{\sigma Q}\right|^{s} \eta^{s}\right)^{1 / s}\left(\int_{\Omega}\left(|D u|^{p(1-1 / s)}+1\right)^{s /(s-1)} \eta^{s(p-1) /(s-1)}\right)^{1-1 / s} \\
& \leq\left(\int_{\Omega}|D u|^{p} \eta^{p}\right)^{1 / p}\left(\int_{\Omega}\left(|D u|^{p}+1\right) \eta^{s(p-1) /(s-1)}\right)^{1-1 / s} \\
& \leq \varepsilon \int_{\Omega}|D u|^{p} \eta^{p}+C(\varepsilon)\left(\int_{\Omega}\left(|D u|^{p}+1\right) \eta^{s(p-1) /(s-1)}\right)^{p(s-1) / s(p-1)} .
\end{aligned}
$$

Hence, combining inequalities (3.4) and (3.5) and choosing $\varepsilon>0$ small enough, we have

$$
\int_{\Omega}|D u|^{p} \eta^{p} \leq C(\varepsilon) \int_{\Omega}\left|u-u_{\sigma Q}\right|^{p}|\nabla \eta|^{p}+C(\varepsilon)\left(\int_{\Omega}\left((D u)^{p}+1\right) \eta^{s /(s-1)}\right)^{p(s-1) / s(p-1)} .
$$

Noticing that $D \eta \leq C|Q|^{-1 / n}$, we obtain

$$
\int_{Q}|D u|^{p} \leq C(\varepsilon)|Q|^{-p / n} \int_{\sigma Q}\left|u-u_{\sigma Q}\right|^{p}+C(\varepsilon)\left(\int_{Q}\left(|D u|^{p}+|u|^{s}+1\right)\right)^{p(s-1) / s(p-1)} .
$$

This completes the proof of Theorem 3.1.

In order to discover the relation between A-harmonic equations and A-Dirac systems, we should remove singularity of solutions to A-Dirac systems at first. Thus, various regularity properties of real-valued functions, such as the following definition [9], are needed.

Definition 3.1 Assume that $u \in L_{\mathrm{loc}}^{1}\left(\Omega, \mathcal{U}_{n}\right), q>0$ and that $-\infty<k \leq 1$. We say that $u$ is of $q, k$-oscillation in $\Omega$ when

$$
\sup _{2 Q \subset \Omega}|Q|^{-(q k+n) / q n} \inf _{u_{Q} \in \mathcal{M}}\left(\int_{Q}\left|u-u_{Q}\right|^{q}\right)^{1 / q}<\infty .
$$

The infimum over monogenic functions is natural since they are trivial solutions to an A-Dirac equation just as constants are solutions to an A-harmonic equation. If $u$ is a function and $q=1$, then (3.7) is equivalent to the usual definition of the bounded mean oscillation when $k=0$ and (3.7) is equivalent to the usual local Lipschitz condition when $0<k \leq 1$ [13]. Moreover, at least when $u$ is a solution to an A-harmonic equation, (3.7) is equivalent to a local order of growth condition when $-\infty<k<0[5,13]$. In these cases, the supremum is finite if we choose $u_{Q}$ to be the average value of the function $u$ over the cube $Q$. It is easy to see that in condition (3.7) the expansion factor ' 2 ' can be replaced by any factor greater than 1 .

If the coefficients of an A-Dirac solution $u$ are of bounded mean oscillation, local Hölder continuous, or of a certain local order of growth, then $u$ is in an appropriate oscillation class [8].

Notice that monogenic functions satisfy (3.7) just as the space of constants is a subspace of the bounded mean oscillation and Lipschitz spaces of real-valued functions. We remark 
that it follows from Hölder's inequality that if $s \leq q$ and if $u$ is of $q$, $k$-oscillation, then $u$ is of $s, k$-oscillation. The following lemma shows that Definition 2.1 is independent of the expansion factor of the cube [9].

Lemma 3.1 Suppose that $F \in L_{\mathrm{loc}}^{1}(\Omega, \mathbb{R}), F>0$ a.e., $\gamma \in \mathbb{R}$, and $\sigma_{1}, \sigma_{2}>1$. If

$$
\sup _{\sigma_{1} Q \subset \Omega}|Q|^{\gamma} \int_{Q} F<\infty
$$

then

$$
\sup _{\sigma_{2} Q \subset \Omega}|Q|^{\gamma} \int_{Q} F<\infty .
$$

Then we can prove the main result.

Proof of Theorem 1.1 Let $Q$ be a cube in the Whitney decomposition of $\Omega \backslash E$.

We use the Whitney decomposition $\mathcal{W}=\{Q\}$ of $\Omega$. The Whitney decomposition consists of closed dyadic cubes with disjoint interiors which satisfy

(a) $\Omega \backslash E=\bigcup_{Q \in \mathcal{W}} Q$,

(b) $|Q|^{1 / n} \leq d(Q, \partial \Omega) \leq 4|Q|^{1 / n}$,

(c) $(1 / 4)|Q|^{1 / n} \leq\left|Q_{2}\right|^{1 / n} \leq 4\left|Q_{1}\right|^{1 / n}$ when $Q_{1} \cap Q_{2}$ is not empty.

Here $d(Q, \partial \Omega)$ is the Euclidean distance between $Q$ and the boundary of $\Omega$ [14].

Thus, if $A \subset \mathbb{R}$ and $r>0$, we can define the $r$-inflation of $A$ as

$$
A(r)=\bigcup_{x \in A} B(x, r) .
$$

From Theorem 3.1, we have

$$
\begin{aligned}
\left(\int_{Q}\left(|D u|^{p}+1\right)\right)^{p(s-1) / s(p-1)} & \leq C\left[\left(\int_{Q}|D u|^{p}\right)^{p(s-1) / s(p-1)}+\left(\int_{Q}\right)^{p(s-1) / s(p-1)}\right] \\
& =C\left[B_{1}+B_{2}\right] .
\end{aligned}
$$

Note that $u \in W^{D, p}(\Omega)$ yields

$$
\begin{aligned}
B_{1} & =\left(|Q| f_{Q}|D u|^{p}\right)^{p(s-1) / s(p-1)}=|Q|^{p(s-1) / s(p-1)}\left(f_{Q}|D u|^{p}\right)^{p(s-1) / s(p-1)} \\
& =C\left(\|u\|_{W^{1, p}}\right)|Q|^{p(s-1) / s(p-1)}
\end{aligned}
$$

and

$$
B_{2}=|Q|^{p(s-1) / s(p-1)} .
$$

Using the Caccioppoli estimate (3.1) and the $p, k$-oscillation condition (3.7), we have

$$
\begin{aligned}
\int_{Q}|D u|^{p} & \leq C \inf _{u_{Q} \in \mathcal{M}_{\sigma Q}}|Q|^{\left.\right|^{\frac{p}{n}}} \int_{\sigma Q}\left|u-u_{\sigma Q}\right|^{p}+C\left(\varepsilon,\|u\|_{W^{1, p}}\right)|Q|^{p(s-1) / s(p-1)} \\
& \leq C|Q|^{a},
\end{aligned}
$$


where $a=(n+p k-p) / n$ and note that $-\infty<k \leq 1$. Since the problem is local (use a partition of unity), we show that (2.13) holds whenever $\phi \in W_{0}^{1, p}\left(B\left(x_{0}, r\right)\right)$ with $x_{0} \in E$ and $r>0$ sufficiently small. Choose $r=(1 / 5 \sqrt{n}) \min \left\{1, d\left(x_{0}, \partial \Omega\right)\right\}$ and let $K=E \cap \bar{B}\left(x_{0}, 4 r\right)$. Then $K$ is a compact subset of $E$. Also, let $W_{0}$ be those cubes in the Whitney decomposition of $\Omega \backslash E$. Notice that each cube $Q \in W_{0}$ lies in $K(1) \backslash K$. Let $\gamma=p(k-1)-k$. First, since $\gamma \geq 1$, it follows that $m(K)=m(E)=0$ [7]. Also, since $a-n \geq \gamma$ using (3.11) and (3.14), we obtain

$$
\begin{aligned}
\int_{B\left(x_{0}, r\right)}|D u|^{p} & \leq C \sum_{Q \in W_{0}}|Q|^{a / n} \leq C \sum_{Q \in W_{0}} d(Q, K)^{a} \leq C \sum_{Q \in W_{0}} \int_{Q} d(x, K)^{a-n} d x \\
& \leq C \int_{K(1) \backslash K} d(x, K)^{a-n} d x \leq C \int_{K(1) \backslash K} d(x, K)^{\gamma} d x<\infty .
\end{aligned}
$$

Hence, $u \in W_{\mathrm{loc}}^{D, p}$.

Next, let $B=B\left(x_{0}, r\right)$ and assume that $\psi \in C_{0}^{\infty}(B)$. Also, let $W_{j}, j=1,2, \ldots$ be those cubes $Q \in W_{0}$ with $l(Q) \leq 2^{-j}$.

Consider the scalar functions

$$
\phi_{j}=\max \left\{\left(2^{-j}-d(x, K)\right) 2^{j}, 0\right\} .
$$

Thus, each $\phi_{j}, j=1,2, \ldots$, is Lipschitz, equal to 1 on $K$ and such that $\psi\left(1-\phi_{j}\right) \in W^{1, p}(B \backslash$ $E)$ with compact support. Hence,

$$
\begin{aligned}
\int_{B}[\overline{A(x, D u)} D \psi-\overline{f(x, D u)} \psi] d x \\
=\int_{B \backslash E}\left[\overline{A(x, D u)} D\left(\psi\left(1-\phi_{j}\right)\right)-\overline{f(x, D u)} \psi\left(1-\phi_{j}\right)\right] d x \\
\quad+\int_{B}\left[\overline{A(x, D u)} D\left(\psi \phi_{j}\right)-\overline{f(x, D u)} \psi \phi_{j}\right] d x,
\end{aligned}
$$

where

$$
\begin{aligned}
& I^{\prime}=\int_{B \backslash E}\left[\overline{A(x, D u)} D\left(\psi\left(1-\phi_{j}\right)\right)-\overline{f(x, D u)} \psi\left(1-\phi_{j}\right)\right] d x, \\
& I^{\prime \prime}=\int_{B} \overline{A(x, D u)} D\left(\psi \phi_{j}\right) d x, \\
& I^{\prime \prime \prime}=\int_{B}-\overline{f(x, D u)} \psi \phi_{j} d x
\end{aligned}
$$

since $u$ is a solution in $B \backslash E, I^{\prime}=0$.

Also, we have

$$
I^{\prime \prime}=\int_{B} A(x, D u) \psi D \phi_{j} d x+\int_{B} \phi_{j} A(x, D u) D \psi d x=I_{1}+I_{2} .
$$


Now there exists a constant $c$ such that $|\psi| \leq c<\infty$. Hence, using Hölder's inequality, we have

$$
\begin{aligned}
\left|I_{1}\right| & \leq C \sum_{Q \in W_{j}} \int_{Q}|A(x, D u)|\left|D \phi_{j}\right| d x \leq C \sum_{Q \in W_{j}} \int_{Q}|D u|^{p-1}\left|D \phi_{j}\right| d x \\
& \leq C \sum_{Q \in W_{j}}\left(\int_{Q}|D u| d x\right)^{(p-1) / p}\left(\int_{Q}\left|D \phi_{j}\right|^{p} d x\right)^{1 / p} .
\end{aligned}
$$

Next, using (3.14), we get

$$
\left|I_{1}\right| \leq C \sum_{Q \in W_{j}}|Q|^{(p(k-1)+n)(p-1) / n p} 2^{j}|Q|^{1 / p} .
$$

Now, for $x \in Q \in W_{j}, d(x, K)$ is bounded above and below by a multiple of $|Q|^{1 / n}$ and for $Q \in W_{j},|Q|^{1 / n} \leq 2^{-j}$. Hence,

$$
\left|I_{1}\right| \leq C \sum_{Q \in W_{j}}|Q|^{-1 / n+1 / p+(p(k-1)+n)(p-1) / n p} \leq C \int_{\cup W_{j}} d(x, K)^{p(k-1)-k} .
$$

Since $\cup W_{j} \subset K(1) \backslash K$ and $\left|\cup W_{j}\right| \rightarrow 0$ as $j \rightarrow \infty$, it follows that $I_{1} \rightarrow 0$ as $j \rightarrow \infty$.

Again, using Hölder's inequality,

$$
\begin{aligned}
\left|I_{2}\right| & \leq C \sup _{B}|D \psi|\left(\int_{\cup W_{j}}|D u|^{p} d x\right)^{(p-1) / p}\left|\cup W_{j}\right|^{1 / p} \\
& \leq C\left(\int_{K \backslash K(1)}|D u|^{p} d x\right)^{(p-1) / p}\left|\cup W_{j}\right|^{1 / p} .
\end{aligned}
$$

Since $u \in W_{\text {loc }}^{1, D}(\Omega)$ and $\left|\cup W_{j}\right| \rightarrow 0$ as $j \rightarrow \infty$, we have that $I_{2} \rightarrow 0$ as $j \rightarrow \infty$. Hence, $I^{\prime \prime} \rightarrow 0$

$$
\begin{aligned}
\left|I^{\prime \prime \prime}\right| & \leq\left(|D u|^{p}+|u|^{s-1}+1\right) \\
& \leq C \sum_{Q \in W_{j}}\left[|Q|^{a}+\int_{Q} C^{\prime} d x\right] \\
& \leq C \sum_{Q \in W_{j}}\left[|Q|^{a}+|Q|\right] \\
& \leq C \sum_{Q \in W_{j}}|Q|^{a} \leq C \int_{\cup W_{j}} d(x, K)^{p(k-1)-k},
\end{aligned}
$$

where we have used the fact that $a=(n+p k-p) / n$ for $-\infty<k \leq 1$.

Since $\cup W_{j} \subset K(1) \backslash K$ and $\left|W_{j}\right| \rightarrow 0$ as $j \rightarrow \infty$, it follows that $I^{\prime \prime \prime} \rightarrow 0$ as $j \rightarrow \infty$. 
Authors' contributions

ZW participated in design of the study and drafted the manuscript. SC participated in conceived of the study and the amendment of the paper. All authors read and approved the final manuscript.

\section{Acknowledgements}

Supported by the National Natural Science Foundation of China (No: 11201415); the Natural Science Foundation of Fujian Province (2012J01027) and Training Programme Foundation for Excellent Youth Researching Talents of Fujian's

Universities (JA12205)

Received: 28 January 2013 Accepted: 12 July 2013 Published: 5 August 2013

\section{References}

1. Abreu-Blaya, R, Bory-Reyes, J, Peña-Peña, D: Jump problem and removable singularities for monogenic functions. J. Geom. Anal., 17(1), 1-13 (2007)

2. Chen, Q, Jost, J, Li, J, Wang, G: Dirac-harmonic maps. Math. Z. 254(2), 409-432 (2006)

3. Chen, Q, Jost, J, Li, J, Wang, G: Regularity theorems and energy identities for Dirac-harmonic maps. Math. Z. 251(1), 61-84 (2005)

4. Chen, Q, Jost, J, Wang, G: Nonlinear Dirac equations on Riemann surfaces. Ann. Glob. Anal. Geom. 33(3), 253-270 (2008)

5. Wang, C: A remark on nonlinear Dirac equations. Proc. Am. Math. Soc. 138(10), 3753-3758 (2010)

6. Wang, C, Xu, D: Remark on Dirac harmonic maps. Int. Math. Res. Not. 20, 3759-3792 (2009)

7. Nolder, CA, Ryan, J: p-Dirac operators. Adv. Appl. Clifford Algebras 19(2), 391-402 (2009)

8. Nolder, CA: Nonlinear A-Dirac equation. Adv. Appl. Clifford Algebras 21(2), 429-440 (2011)

9. Nolder, CA: A-harmonic equations and the Dirac operator. J. Inequal. Appl. 2010, Article ID 124018 (2010)

10. Kaufman, R, Wu, JM: Removable singularities for analytic or subharmonic functions. Ark. Mat. 18(1), 107-116 (1980)

11. Koskela, $\mathrm{P}, \mathrm{Martio}, \mathrm{O}$ : Removability theorems for solutions of degenerate elliptic partial differential equations. Ark. Mat. 31(2), 339-353 (1993)

12. Kilpelainen, $T$, Zhong, $X:$ Removable sets for continuous solutions of quasilinear elliptic equations. Proc. Am. Math. Soc. 130(6), 1681-1688 (2002)

13. Meyers, NG: Mean oscillation over cubes and Hölder continuity. Proc. Am. Math. Soc. 15(5), 717-721 (1964)

14. Stein, EM: Singular Integrals and Differentiability Properties of Functions. Princeton Mathematical Series, vol. 30. Princeton University Press, Princeton (1970)

doi:10.1186/1029-242X-2013-362

Cite this article as: Wang and Chen: The relation between A-harmonic operator and A-Dirac system. Journal of Inequalities and Applications 2013 2013:362.

\section{Submit your manuscript to a SpringerOpen ${ }^{\ominus}$ journal and benefit from:}

- Convenient online submission

- Rigorous peer review

- Immediate publication on acceptance

Open access: articles freely available online

- High visibility within the field

- Retaining the copyright to your article 